\title{
GREATLY ENHANCED DETECTABILITY OF GEOTHERMAL TRACERS THROUGH LASER-INDUCED FLUORESCENCE
}

\author{
Final Technical Report for DOE Project DE-FG07-98ID13619
}

\author{
October 30, 2002 \\ ${ }^{1}$ Peter E. Rose, Principal Investigator \\ ${ }^{2}$ Joel Harris, Co-Principal Investigator, Professor \\ ${ }^{1}$ Phaedra Kilbourn, Research Chemist \\ ${ }^{2}$ James Kleimeyer, Research Assistant \\ ${ }^{1}$ Troy Carter, Research Assistant \\ ${ }^{1}$ Energy and Geoscience Institute, University of Utah \\ ${ }^{2}$ Department of Chemistry, University of Utah
}

\subsection{Summary of Progress}

We have successfully completed a four-year R\&D project to greatly reduce the detection limit of fluorescent tracers through the use of emerging laser-excitation, optical fiber, and CCDspectroscopy technologies. Whereas the efforts over the first two years were directed at demonstrating a reduction in the detection limit of fluorescent compounds by a factor of 100 and at identifying several new fluorescein-derived tracer candidates, our recent efforts were focused primarily on the field demonstration of new tracers having detection limits in the low parts-perquadrillion range. During the summer of 2001, we initiated field tests at the Dixie Valley, Nevada and at the Beowawe, Nevada geothermal fields using very small quantities of the fluoresceinderivative 6-carboxyfluorescein. Subsequently, we succeeded in measuring sub-part-per-trillion quantities of that candidate tracer at both the Beowawe and Dixie Valley geothermal reservoirsusing approximately $530 \mathrm{~g}$ of tracer at each setting. Our studies indicate that we could have observed breakthrough using only $0.53 \mathrm{~g}$ of 6-carboxyfluorescein. This represents a reduction by a factor of 170,000 below the mass of tracer used in a previous tracer test at Beowawe.

\subsection{Background Summary and Objectives}

Prudent reservoir management strategies invariably require the reinjection of produced fluids into geothermal reservoirs via dedicated injection wells. If the injection wells are located too close to the production wells, there exists a risk of premature thermal breakthrough. If the injectors are placed to far from the producers, then the injectate will not return to the reservoir, and the result will be a drop in reservoir pressure. Thermally stable and detectable organic tracers are needed within the geothermal industry in order to optimize injection well placement.

Whereas fluorescent compounds are among the most detectable tracers used in the geothermal industry, hundreds of kilograms are still typically required for each injection well that is tagged during a tracer test. As detection limits are lowered, however, the amount of tracer required is 
proportionally reduced, with a concomitant reduction in tracer cost. Likewise, since a chemically distinct tracer is required for each injector that is tagged, many tracers are required in order to tag simultaneously all of the injectors within a reservoir. And, since most candidate fluorescent tracers are not manufactured in bulk, an increase in detectability results in an expansion of the list of candidate tracers. The objective of this research project is to increase the detectability of fluorescent tracer candidates by a factor of 100. This will result in not only a great reduction in the cost of performing a tracer test, but also a significant increase in the number of candidate tracer compounds available. Also, many of these technologies are relevant to the development of rugged, affordable, on-line tracer detection at the well head, which geothermal operators have long desired.

Our experimental approach has developed along two general pathways. The first was the design, fabrication, and evaluation of the analytical equipment, based upon the concepts of laser-induced fluorescence. Second has been the selection and evaluation of candidate fluorescent tracer compounds. These compounds are sufficiently similar to fluorescein that they require the same excitation wavelength as fluorescein, serving to greatly simplify the analytical apparatus.

\subsection{Equipment Fabrication, and Testing}

With the advent of intense laser light sources and very efficient charge-coupled-device (CCD) spectrometers, it is possible to greatly enhance the detectability of fluorescent tracers. In addition, conventional high performance liquid chromatography (HPLC) allows for the efficient separation of fluorescent tracers not only from each other but also from naturally fluorescing contaminants present in geothermal reservoir water. Shown in Figure 1 is a schematic drawing of an HPLC/laser-fluorimeter assembly that was designed and fabricated in our laboratory.

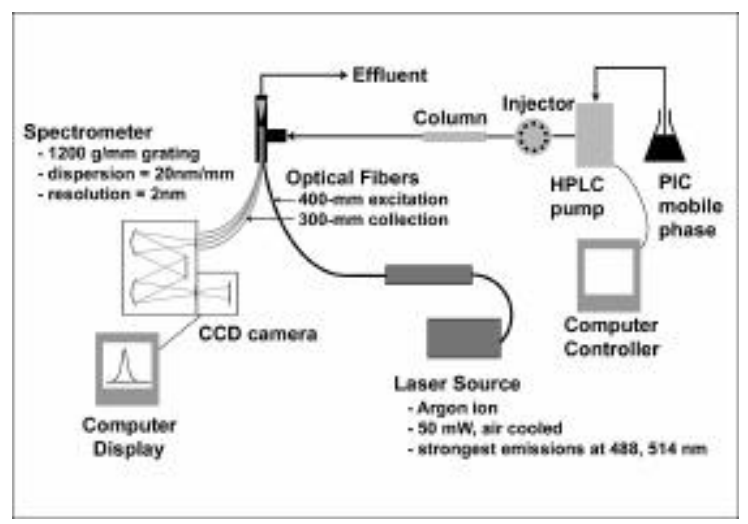

Figure 1. The HPLC/laser fluorimeter assembly.

One of the advantages of the fluorimeter shown in Figure 1 is that the CCD detector allows for the collection of an entire fluorescence emission spectrum with each chromatogram. The raw emission spectra for fluorescein at concentrations between 50 parts per quadrillion and 750 parts per quadrillion are plotted as functions of wavelength in Figure 2. By integrating over the entire wavelength for each curve at each time step, the chromatograms in Figure 3 were created. 




Figure 2. Raw CCD spectra for fluorescein between 50 and 750 parts per quadrillion.

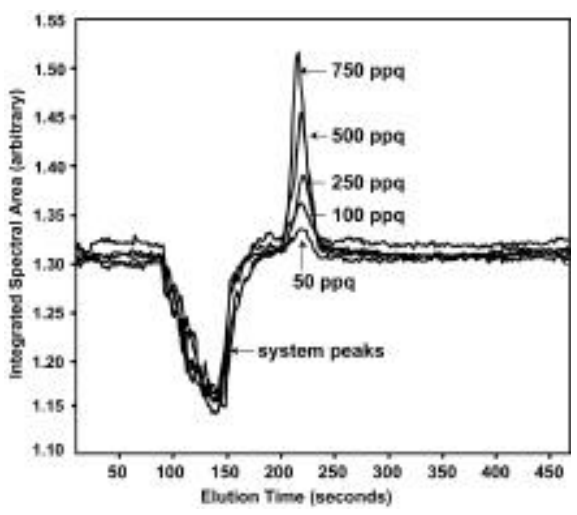

Figure 3. Chromatograms of fluorescein solutions between 50 and 750 parts per quadrillion obtained by integrating over the entire emission spectrum of each solution.

Finally, by integrating each curve in Figure 3, the calibration curve in Figure 4 was obtained, indicating that a reasonably linear relationship between concentration and fluorescence emission can be obtained over this concentration range. The detection limit for fluorescein was determined to be $40 \mathrm{ppq}$, which represents an increase in detectability over conventional HPLC instruments by a factor of approximately 100 (Kleimeyer et al., 2001).

Fluorescein has been extensively used as a tracer in hydrothermal systems. Not only is it very detectable by conventional fluorescent spectroscopy techniques, but it is also quite thermally stable and can be used in geothermal reservoirs as hot as about $250^{\circ} \mathrm{C}$ (Adams and Davis, 1991). Several derivatives of fluorescein have recently been synthesized for biochemical applications. We have therefore evaluated this family of compounds with the expectation that some of the fluorescein derivatives would possess similar properties of thermal stability and detectability (Wong and Rose, 2000; Wong, 2000). 


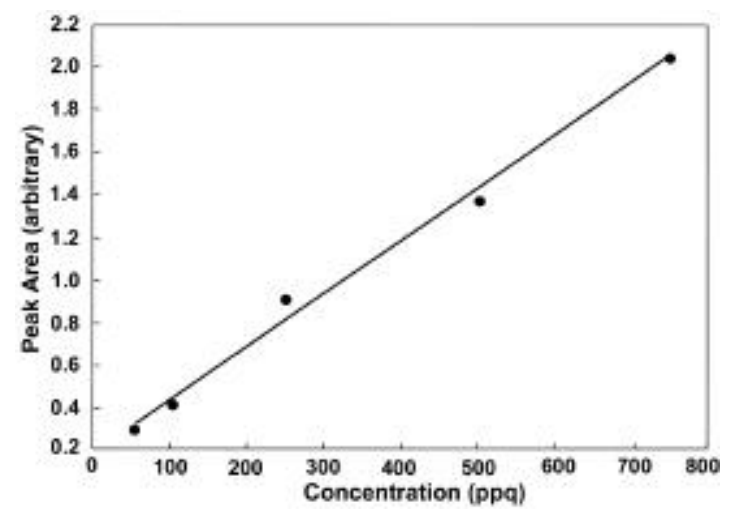

Figure 4. Calibration curve for fluorescein showing a linear relationship between concentration and peak area over the range of interest.

Nine candidate tracers were identified from among the fluorescein derivatives. Those nine were screened by exposing them to conditions that simulate a hydrothermal environment in laboratory reactors at a temperature of $270^{\circ} \mathrm{C}$ for 48 hours. Of the nine, four were shown to possess reasonably good thermal stability. The residual undecayed percentage for the four most stable compounds is shown in Figure 5. For comparison, fluorescein is also shown. It is evident from the figure that one of the compounds, 6-carboxyfluorescein is comparable in stability to fluorescein. We therefore selected this compound for a detailed thermal stability study in anticipation of its use in a field test.



Figure 5. The structural formula for fluorescein and four fluorescein derivatives and their residual undecayed percentages upon exposure to geothermal conditions for two days at $270^{\circ} \mathrm{C}$.

The thermal decay kinetics of 6-carboxyfluorescein was modeled by the first-order differential equation (Wong, 2000; Wong and Rose, 2000):

$$
? d C_{c} / d t ? k_{c} ? C_{c}
$$


where $\mathrm{C}_{\mathrm{c}}$ is the concentration of naphthalene sulfonate and $\mathrm{k}_{\mathrm{c}}$ is the first-order rate constant. Solution of this equation results in the following relationship between $\mathrm{C}_{\mathrm{c}}$ and $\mathrm{t}$ :

$$
\ln \left\{\frac{C_{c}}{C_{c}^{0}} ? ? ? \text { ? } k_{c} ? t\right.
$$

where $\mathrm{C}_{\mathrm{c}}^{0}$ is the initial concentration of polyaromatic sulfonate. The temperature dependence of $\mathrm{k}_{\mathrm{c}}$ can be described by the Arrhenius relationship:

$$
k_{c} ? A e^{? ? E_{a} / R T ?}
$$

where $\mathrm{A}$ is the pre-exponential factor, $\mathrm{E}_{\mathrm{a}}$ is the energy of activation, $\mathrm{R}$ is the gas constant and $\mathrm{T}$ is absolute temperature. A linearization of the Arrhenius expression results in the following:

$$
\ln k_{c} ? \ln A ? \frac{E_{a}}{R T}
$$

Figure 6 shows an Arrhenius plot of $\ln \mathrm{k}_{\mathrm{c}}$ vs. inverse temperature between $270^{\circ} \mathrm{C}$ and $290^{\circ} \mathrm{C}$ for 6 carboxyfluorescein. A fairly linear relationship between $\ln \mathrm{k}_{\mathrm{c}}$ and inverse temperature was observed, indicating that the Arrhenius equation provides a reasonably good means of expressing the temperature dependence of the decay rate constant. The constants $\mathrm{A}$ and $\mathrm{E}_{\mathrm{a}}$ were determined to be $7.54 \mathrm{E} 4 \mathrm{~s}^{-1}$ and $1.11 \mathrm{E} 5 \mathrm{~J} / \mathrm{mol}$, respectively.



Figure 6. Arrhenius plot for 6-carboxyfluorescein based upon first-order decay rate kinetics.

\subsection{Field Campaigns}

In order to test the greatly enhanced detectability of the laser fluorimeter, we studied the candidate tracer 6-carboxyfluorescein in tracer tests at the Beowawe and Dixie Valley geothermal systems.

\section{Beowawe Tracer Test}

On July 12, 2001, $530 \mathrm{~g}$ of 6-carboxyfluorescein was injected into well 85-18 at the Beowawe 
(Caithness Corporation) geothermal field (Figure 7). The injector was flowing at approximately

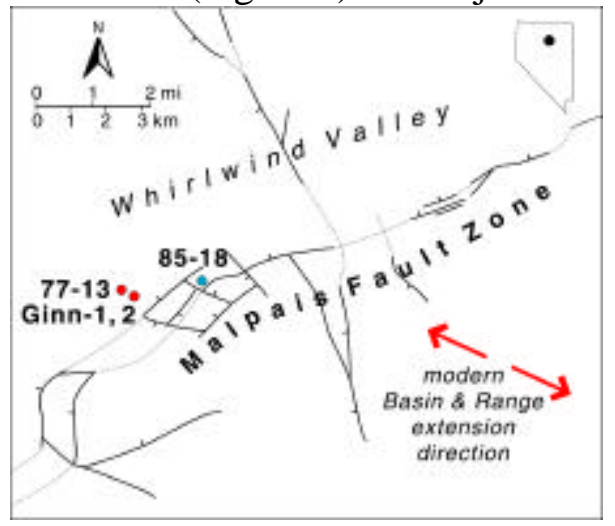

Figure 7. A plan view of the Beowawe geothermal reservoir, showing the injection (85-18) and production (Ginn 1, Ginn 2, and 77-13) wellhead locations.

$220 \mathrm{~kg} / \mathrm{sec}$. The reservoir's three production wells were subsequently monitored and analyzed for 6-carboxyfluorescein using conventional HPLC methods over the subsequent several months.

The tracer test in 2001 was not the first at Beowawe involving injector 85-18. Almost seven years to the day (July 13, 1994), $91 \mathrm{~kg}$ of fluorescein was mixed with approximately $23 \mathrm{~m}^{3}$ of reservoir water and injected as a slug at a rate of approximately $220 \mathrm{~kg} / \mathrm{sec}$ into $85-18$ (Rose et al., 1995). The surrounding production wells were sampled and analyzed for fluorescein over the subsequent several years.

Figure 8 shows the return of the tracers to the reservoir's three production wells (Ginn 1, Ginn 2, and 77-13) for each test. In both cases, the returns were normalized by dividing by the mass of tracer injected. The curves do not overlie each other perfectly, which may result from an incorrect knowledge of the mass of tracer injected—at least in the first test. In the 1994 test, $91 \mathrm{~kg}$ of fluorescein was mixed with produced brine in a 6,000-gal tank prior to injection into the reservoir. An unknown volume of tracer solution was left in the tank, but the normalized return curve shown in Figure 8 was based on a 91-kg injection mass.

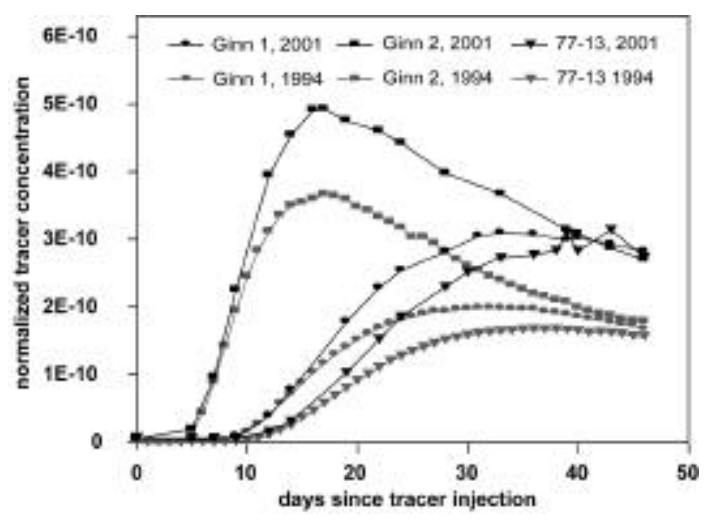


Figure 8. Normalized returns for the 1994 and 2001 tracer tests at the Beowawe geothermal field.

In the case of the 2001 test, the mass of tracer was measured quite accurately as $530 \mathrm{~g}$ and essentially all of the $530 \mathrm{~g}$ of 6-carboxyfluorescein was injected. Therefore, the normalized values shown for 6-carboxyfluorescein for the 2001 test are considered to be more accurate than the normalized fluorescein values for the 1994 test. Regardless of the differences, the return curves for each well pair are quite similar with first arrivals and peak maxima almost identical, indicating that reservoir flow patterns changed very little during the seven years between tests.

The tracer-return data shown for the 2001 test in Figure 8 were obtained using HPLC with conventional fluorescence detection. After analyzing the tracer-return data, we observed that much less tracer than the amount used would have been necessary to measure tracer breakthrough at Beowawe's three production wells. In order to demonstrate this, we diluted the samples by a factor of 1,000 and reanalyzed them for 6-carboxyfluorescein using the HPLC with LIF detection. Shown in Figure 9 are plots of tracer concentration both for undiluted Ginn-2 samples, as measured by conventional HPLC, and diluted Ginn-2 samples, as measured by HPLC with LIF detection. The curves do not overlie each other perfectly but reproduce each other reasonably well. This demonstrates that only one thousandth as much tracer $(0.53 \mathrm{~g}$ of 6carboxyfluorescein) would have been required to produce measurable breakthrough. This represents a reduction in tracer mass by a factor of approximately 170,000 compared to the quantity used in the 1994 test!



Figure 9. Undiluted and diluted tracer returns to Beowawe producer Ginn 2, analyzed using conventional fluoresence and laser-induced fluorescence detection methods, respectively.

\section{Dixie Valley Tracer Test}

The Dixie Valley geothermal system presents a more challenging hydrothermal environment for organic tracers than the Beowawe reservoir. Not only are the fluid temperatures somewhat hotter, but the interwell residence times are significantly longer. On July 10, 2001, $530 \mathrm{~g}$ of 6carboxyfluorescein was injected into well 38-32 at the Dixie Valley (Caithness Corporation) geothermal field (Figure 10). Simultaneously, $150 \mathrm{~kg}$ of the candidate tracer 2,6-naphthalene disulfonate was injected as a companion tracer into the same well, which was flowing at about 
$110 \mathrm{~kg} / \mathrm{sec}(1650 \mathrm{gpm})$. All of the reservoir's production wells were sampled and analyzed for 2,6-naphthalene disulfonate during the subsequent several months using conventional HPLC techniques (Rose et al., 2002).

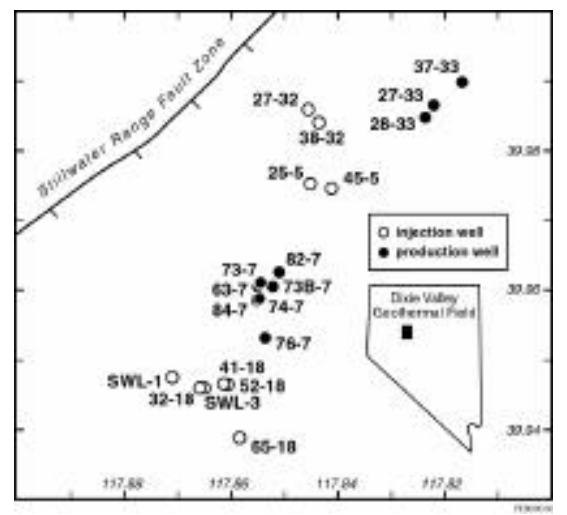

Figure 10. A plan view of the Dixie Valley geothermal reservoir, showing the injection and production wellhead locations.

Shown in Figure 11 are the returns of the two tracers to well 28-33. The values in this figure were normalized by dividing the measured concentrations by the mass of each tracer injected. If both tracers behaved conservatively, their normalized return curves would overlie each other. It is evident that the 6-carboxyfluorescein values are considerably lower than those of the conservative tracer, 2,6-naphthalene disulfonate, indicating that this tracer was extensively thermally degraded as it traveled through the reservoir. Also shown in Figure 11 is the corrected return curve for 6-carboxyfluorescein. The points on this curve were calculated based upon the thermal decay kinetics reported in this paper, assuming an effective temperature of $217^{\circ} \mathrm{C}$. This temperature is between the approximate bottom-hole temperatures of $200^{\circ} \mathrm{C}$ and $242^{\circ} \mathrm{C}$ for the injection well (38-32) and production well (28-33), respectively.



Figure 11. Return of tracers 2,6-naphthalene disulfonate and 6-carboxyfluorescein from well 38-32 to well 28-33 at the Dixie Valley geothermal reservoir. The temperature-corrected 6- 
carboxyfluorescein curve was calculated based upon the measured decay kinetics of 6carboxyfluorescein, assuming an effective temperature of $217^{\circ} \mathrm{C}$.

\subsection{Summary and Conclusions}

All of the initial programmatic objectives of this project were achieved. A highly sensitive laser fluorimeter was designed and fabricated, based upon state-of-the-art fiber optics, laser light sourcing and CCD spectrometry. Using this detector, the detection limit for fluorescein was reduced to $40 \mathrm{ppq}$, representing an improvement in sensitivity by a factor of 100 over conventional fluorescence detection.

A family of fluorescent compounds was identified for its potential in geothermal tracing applications. Four compounds from this family were screened for thermal stability and found to be comparable to fluorescein and acceptable for use in intermediate-temperature reservoirs. One compound from this family, 6-carboxyfluorescein, was selected for extensive thermal stability analysis in order to determine its decay kinetics and Arrhenius parameters.

In order verify the laboratory experiments, the candidate tracer 6-carboxyfluorescein was tested at the Beowawe and Dixie Valley geothermal reservoirs. In each test approximately one half kilogram of the tracer was injected at the target injectors and monitored over several months in the surrounding production wells. The tests showed that the mass of tracer injected could be reduced by a factor of more than 100, verifying the reduction in detection limit by a factor of 100 .

This project demonstrated that the mass of tracer typically used in a geothermal tracer test could be significantly reduced. Likewise, many fluorescent compounds that are not manufactured in bulk could be added to the list of candidate fluorescent tracers, since only a small quantity would be required for each test.

\section{References}

Kleimeyer, J.A., Rose, P.E., and Harris, J.M., Determination of ultratrace-level fluorescent tracer concentrations in environmental samples using a combination of HPLC separation and laserexcited multiwavelength fluorescence emission detection: application to testing of geothermal well brines: Applied Spectroscopy, 55(6), 690-700.

Rose, P.E., Adams, M.C., and Benoit, W.R. (1995) A tracer test at the Beowawe geothermal field, Nevada, using fluorescein and tinopal CBS: Geothermal Resource Council Transactions, 19, 217-221.

Rose, P.E., Johnson, S.D., and Kilbourn, P.M., and Kasteler, C. (2002) Tracer Testing at Dixie Valley, Nevada Using 1-Naphthalene Sulfonate and 2,6-Naphthalene Disulfonate: Proc. TwentySixth Workshop on Geothermal Reservoir Engineering, Stanford University, SGP-TR-171. 
Wong, Y.L., 2000, An evaluation of fluorescein-derived compounds for use as geothermal tracers: M.S. thesis, Department of Chemical and Fuels Engineering, University of Utah.

Wong, Y.L. and Rose, P.E., The testing of fluorescein derivatives as candidate geothermal tracers: Geothermal Resources Council Transactions, 24, 637-640. 